\title{
Qui décide de ma vie et de ma mort?
}

\author{
Jean Martin \\ Dr med., membre de la rédaction
}

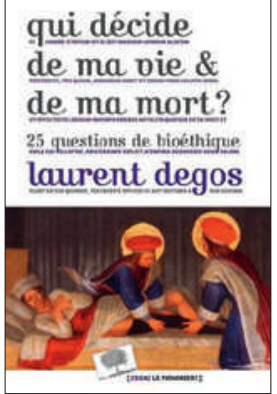

Laurent Degos Qui décide de ma vie et de ma mort?

25 questions de bioéthique Paris: Le Pommier; 2015. 90 pages. $18.60 \mathrm{CHF}$. ISBN 978-2-746-50934-4
Laurent Degos est un hématologue et immunologue parisien. Après d'autres ouvrages, il publie un essai bref sur les enjeux actuels de bioéthique, regroupés en quatre parties: Qu'est-ce qu'une personne? (et depuis quand); Qui décide de la mort? (y compris problématique du suicide assisté); La dignité de la personne (le consentement est-il toujours libre?); A qui appartient le corps? (notamment à propos de don d'organes et transplantation). Dans son avant-propos: «L'éthique est la conscience de ce qui est bien ou mal; c'est une science morale qui n'est pas censée varier avec le temps ni même être mise en forme dans des lois». Vision plutôt globalisante, fondamentale, de l'éthique? Pourtant, il ajoute: "[Mais] comment définir les frontières du bien et du mal dans une discipline, la biomédecine, qui ne cesse d'évoluer? [...] Or, au quotidien, les praticiens doivent examiner toutes les issues possibles pour chaque cas et proposer la solution la mieux adaptée au moment, à la culture et à la connaissance scientifique». Ici se marque une approche plus contextuelle et relative de la bioéthique. Degos se distancie donc de positions qui voudraient que de nombreux éléments de doctrine soient absolus, ne puissent jamais être remis en question, alors même que la réalité vécue dans nos sociétés évolue de manière majeure - et rapide. Exprimant ainsi cette ouverture: «Les lois bioéthiques oscillent entre un accès au corps biologique [de diverses façons] et la protection de la personne. A ce titre, les lois et avis bioéthiques ne sont ni universels ni pérennes, car ils dépendent de la culture de la société qui les émet.»

Dans cet ouvrage ramassé, il aborde beaucoup des défis actuels pour la pratique biomédicale, qui demandent des appréciations sociétales et soulèvent la question d'élaborations juridiques. Il les traite en plantant le décor de chacun (y compris les dispositions légales actuelles) et en indiquant des options possibles, mais le plus souvent sans porter de jugement ni donner de réponse. Ce qui pourra surprendre le lecteur qui serait venu trouver des solutions... Mais le fait est qu'il est surtout nécessaire, aujourd'hui, d'ouvrir l'esprit aux avancées impressionnantes des sciences et des techniques et de proposer des moyens de les apprécier, sans vouloir à chaque fois formuler une conclusion qui pourra s'avérer rapidement obsolète. Et Degos de noter que son ouvrage contient les éléments utiles aux élèves de classe terminale pour leur cours d'éducation civique, juridique et sociale. Il évite l'écueil de donner des conseils de façon paternaliste mais peut-être aurait-il pu néanmoins tracer un ou quelques chemins d'élaboration des thèmes traités?

Evoquant le fait que l'innovation implique souvent un conflit éthique, il pose la question «Faut-il promouvoir le progrès (intérêt collectif) ou protéger la personne (intérêt individuel)?», qui illustre la tension entre santé publique et santé individuelle. Il cite l'Avis no 73 de 2002 du Comité consultatif national d'éthique disant: "Le progrès médical s'est souvent fondé sur des rapports bénéfices/risques initialement asymétriques [...] La société dans son ensemble doit être consciente que l'exigence de la recherche peut conduire à privilégier parfois les intérêts de la communauté». Tout en relevant que les pesées d'intérêts sont particulièrement ardues dans les situations qu'il appelle sauts technologiques.

L'espèce humaine est riche de sa diversité et de sa vulnérabilité. Le rejet du vulnérable amène à la barbarie.

Dans son interpelante conclusion: «L'espèce humaine est riche de sa diversité, qui lui permet de survivre face à tout type d'agression, riche de sa vulnérabilité, qui permet de révéler sa solidarité. L'homogénéité signe sa mort. Le rejet du vulnérable et du fragile amène l'humanité à la barbarie. Le désir de la normalité et la quête d'un transhumanisme sont deux grandes tentations, rendues envisageables par la science, mais aussi deux impasses». Beau programme de réflexion éthique, $\mathrm{au}$ moment où congrès et revues spécialisées sont pleins d'envolées trans- ou post-humanistes - et où, dans la pratique quotidienne, beaucoup d'efforts de la médecine (des obstétriciens, pédiatres, généticiens et d'autres) tendent à assurer que les enfants qui naissent et les adultes qu'ils deviendront soient aussi bien (parfaits?) que possible. Qui arrêtera ce train? Peut-on/ faut-il l'arrêter ou contrôler sa direction et sa vitesse? 